\title{
Emergent Properties of Antiagglomerant Films Control Methane Transport: Implications for Hydrate Management
}

\author{
François Sicard ${ }^{1}$. Tai Bui ${ }^{1}$, Deepak Monteiro ${ }^{2}$, Qiang Lan $^{2}$, Mark Ceglio ${ }^{2}$, Charlotte Burress ${ }^{2}$, and Alberto Striolo ${ }^{1}$ \\ ${ }^{1}$ Department of Chemical Engineering, University College London, WC1E rJE, London, UK and \\ 2 Halliburton, Houston, Texas, USA
}

\begin{abstract}
The relation between collective properties and performance of antiagglomerants (AAs) used in hydrate management is handled using molecular dynamics simulations and enhanced sampling techniques. A thin film of AAs adsorbed at the interface between one flat sII methane hydrate substrate and a fluid hydrocarbon mixture containing methane and $n$-dodecane is studied. The AA considered is a surface-active compound with a complex hydrophilic head that contains both amide and tertiary ammonium cation groups and hydrophobic tails. At sufficiently high AA density, the interplay between the surfactant layer and the liquid hydrocarbon excludes methane from the interfacial region. In this scenario, we combine metadynamics and umbrella sampling frameworks to study accurately the free-energy landscape and the equilibrium rates associated with the transport of one methane molecule across the AA film. We observe that local configurational changes of the liquid hydrocarbon packed within the AA film are associated with high free-energy barriers for methane transport. The time scales estimated for the transport of methane across the AA film can be, in some cases, comparable to those reported in the literature for the growth of the hydrates, suggesting that one possible mechanism by which AAs delay the formation of hydrate plugs could be providing a barrier to methane transport. Considering the interplay between structural design and collective properties of AAs might be of relevance to improve their performance in flow assurance.
\end{abstract}

\section{INTRODUCTION}

Gas hydrates, also known as clathrate hydrates, are ice-like inclusion compounds consisting of polyhedral hydrogen-bonded water cages stabilized by guest gas molecules [1 $[3]$. They are not chemical compounds because no strong chemical bonds exist between water and gas molecules [4, 5]. They are formed under high-pressure and low-temperature conditions such as those found in deep oceans and pipelines [6]. The gas molecules able to be trapped (enclathrated) into the water cages are usually small $(<10 \AA)$ : methane, ethane, propane, 1-butane, nitrogen, hydrogen, and carbon dioxide [7]. Clathrate hydrates are relevant in a variety of scientific and industrial contexts, including climate change modeling 8], carbon dioxide sequestration [9], hydrocarbon extraction [10], hydrogen and natural gas storage 10-12], separation and refrigeration technologies [13], marine biology 14], and planetary surface chemistry [15]. Of particular interest are the hydrocarbon hydrates that can form blockages in oil and gas pipelines [3, 16]. This phenomenon severely affects the safety of pipeline flow assurance [16].

Three major stages are associated with hydrate plug formation: nucleation [3, 17], growth [3, 18], and agglomeration 19, 20]. Numerous experimental investigations [3, 21 26], modeling, and simulations 27 31] have contributed to the current understanding of such stages. As offshore drilling activities have moved towards geological sites of deeper waters and colder temperatures [32], the community is facing ever-more severe technical challenges. To manage hydrates in pipelines, hydrate inhibitors are used. They are differentiated depending on their mode of action: thermodynamic hydrate inhibitors (TIs) 10, such as methanol and monoethylene glycol, shift the stability conditions of hydrates to lower temperatures and higher pressures, but require large amounts (10 to $50 \mathrm{wt} \%$ ) to be effective. Low dosage hydrate inhibitors (LDHIs) 2] instead are effective at concentrations as low as $1 \mathrm{wt} \%$ of water. LDHIs were introduced in the mid-1980s and early 1990s 33]. Unlike TIs, LDHIs do not influence the thermodynamics of hydrates formation but affect its kinetics 2]. Kinetic hydrate inhibitors (KHIs) and antiagglomerants (AAs) are the two main LDHIs classes 34]. Most KHIs are polymeric compounds containing amide groups, such as poly( $N$-vinylpyrrolidone), polyvinylcaprolactam, and polydiethylacrylamide 2, 35]. They are believed to delay hydrate nucleation and/or growth. AAs, mostly surface-active surfactants, are usually amphiphilic chemicals with complex hydrophobic tails and hydrophilic headgroups [36]. They allow the hydrate particles to form but keep them dispersed, yielding transportable slurries [2, 16, 37]. When AAs adsorb at the oil-hydrate interface, the hydrophobic tails preferably point toward the hydrocarbon phase, possibly inducing an effective repulsion when two hydrates approach each other [3, 38, 39]. When the AAs polar headgroups are adsorbed on the hydrate surface, they could interfere with the hydrate growth 37, 40]. Quaternary ammonium salts, first developed by Shell in the early 1990s, are the most well-known AAs [4]. Promising performance of commercial additives and new AAs have been reported by several research groups [37, 42 46]. Most AAs are only effective at low water content (e.g., less than $30 \%$ ), 
but some can be effective at high water content (up to $80 \%$ ), such as those reported by Gao [47]. While the use of AAs is increasing in subsea projects across the industry [48], their mechanisms of action remain poorly understood. Such understanding is necessary to improve their cost effectiveness and expand the range of conditions over which their use is safe and convenient.

Because classical molecular dynamics (MD) simulations can follow the trajectories of individual molecules, MD has been the preferred technique to investigate the formation of hydrates with and without the presence of KHIs [4, 5, 36, 39, 49 51]. Recent numerical studies have concentrated on the relation between structure and performance of model AAs, with the emergent molecular-level characterization of the surface adsorption mechanisms of surfactants to hydrates considered as a signature of microscopic performance [52, 53]. The coalescence mechanisms of gas hydrate crystals and water droplet have also been studied [39]. Recently, Bui et al. [36] related the macroscopic performance of a class of AAs in flow-assurance applications to the molecularlevel properties of the surfactant interfacial film. Those simulations, compared to experiments, suggested that effective AAs could provide energy barriers in methane transport.

In the present work, the authors quantify such energy barriers as experienced by one methane molecule diffusing from the hydrocarbon phase to the growing hydrate. Building on the work of Bui et al. [36], we consider the AA that is most effective at excluding methane from the film of AAs formed at the water-hydrocarbon interface. This AA was determined to have good performance in laboratory tests designed to screen AAs for flow assurance applications. The metadynamics 54] (metaD) and umbrella sampling [55] (US) frameworks are combined to study accurately the free-energy (FE) landscape and the equilibrium rates associated with the transport mechanisms of one free methane molecule across a densely packed interfacial layer. At sufficiently high AA density, we show that the FE barrier is caused by local configurational changes of the liquid hydrocarbon molecules packed within the AA film.

\section{RESULTS AND DISCUSSION}

\section{Interfacial structure}

The molecular structure of the AA considered in this study is shown in Fig. 1 with $R_{1}$ and $R_{2}$ groups representing long and short tails in one AA, respectively. The AA molecule headgroup includes both amide and tertiary ammonium cation groups. Following the notation of Bui et al. [36], the AA are denoted as $S_{X} L_{Y}$,

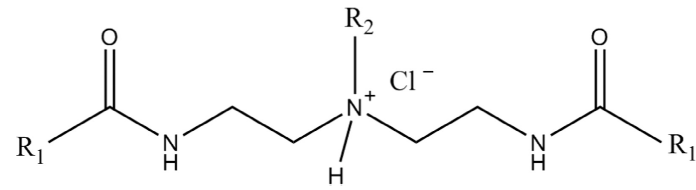

FIG. 1: Molecular structure of the AA with two long tails $R_{1}$ (n-dodecyl) and one short tail $R_{2}$ ( $n$-butyl).

where $X=4$ ( $n$-butyl) and $Y=12$ ( $n$-dodecyl) indicate the number of carbon atoms in the short $(\mathrm{S})$ and long (L) tail, respectively. The long AA tails become ordered, almost perpendicular to the hydrate surface, when the surface coverage increases, as previously reported by Bui et al. [36]. We consider a surface density $\approx 0.67$ molecule $/ \mathrm{nm}^{2}$, sufficiently high to yield a dense ordered film at the hydrate-hydrocarbon interface and sufficiently low to allow interface regions with both low and high AA density. To define unambiguously the spatial organization of the AA layer, the position of the central Nitrogen atom on each surfactant in Fig. 2 was fixed using a restraint potential, as implemented in GROMACS [56]. This additional numerical constraint does not impact the analysis of the transport properties of the free methane molecule through the AA layer as the AAs remain adsorbed at the hydrate-hydrocarbon interface within the timeframe of our MD simulations. The initial configuration used in the simulations is shown in Fig. 2 .

To characterize the thermodynamic and dynamical properties of the AA film adsorbed at the interface between the flat sII methane hydrate and the hydrocarbon fluid, one must consider the non-isotropic distribution of the surfactant film at the interface. This spatial distribution depends on the AA concentration 36] and yields different pathways available to the passage of the free methane molecule. We quantified the efficiency of the AA layer in limiting the transport of the methane molecule with the identification of the minimal FE pathway. This analysis allowed for study of the transport mechanisms at play when one free methane molecule travels through the interface.

\section{Identification of representative pathways}

To identify representative pathways available to the methane molecule to cross the AA film, we ran WTmetaD simulations, as discussed previously. This approach was necessary to handle the non-isotropic distribution of the AAs within the interfacial layer. However, it did not prevent hysteresis effects caused by local reorganization of the liquid hydrocarbons within the AA layer. This hysteresis effect might occur when the bias potential becomes sufficiently high to expel hydrocarbon molecules from the interfacial layer. To prevent this possible shortcoming, one should consider an additional CV, 
accounting for the local behaviour of the liquid hydrocarbon molecules. This is well known, for instance, in biological docking studies 5760 . Computational limitations made it unfeasible to consider an additional $\mathrm{CV}$ in the present work.

Instead, we ran WT-metaD simulations to identify representative pathways. Figure 3 shows representative FEPs obtained within the WT-metaD framework, along with the respective snapshots to illustrate the different scenarios. The pathways identified occur in interfacial regions with different AA density. The minimal FEP corresponds to the diffusion of the methane through an interfacial region made up of a large cluster of hydrocarbons. These regions show a characteristic size $\approx 20 \AA$ in the simulations, which is comparable to the molecular dimension of the dodecane molecules (cf. Figure 3 a). When hydrocarbons are confined in these narrow regions, it is possible that the effective viscosity differs with respect to that in the bulk 61]. The intermediate FEP corresponds to the diffusion of the methane along the edge of a hydrocarbon cluster to the surrounding AA molecules. As shown in Figure 3b, the methane molecule

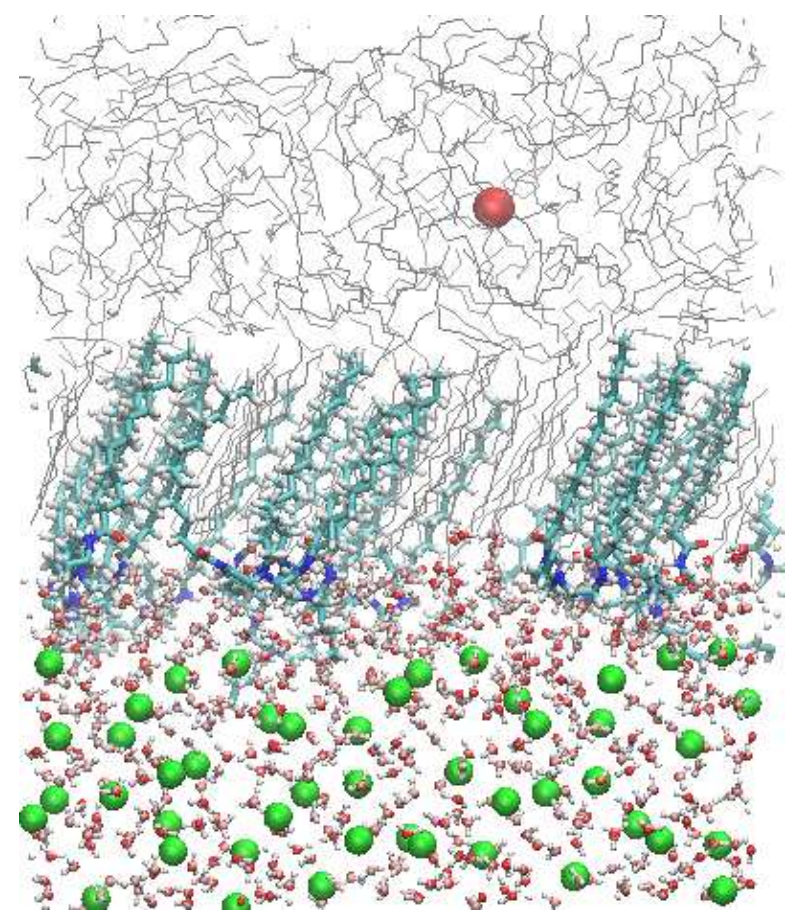

FIG. 2: Initial configuration obtained after equilibration for an AA surface density $\approx 0.67$ molecule $/ \mathrm{nm}^{2}$. Green spheres represent methane molecules in the sII methane hydrate. Grey lines represent $n$-dodecane molecules, either in the bulk or trapped within the AA layer. The free methane molecule is shown as a red sphere. Yellow, red, blue, white, and cyan spheres represent chloride ions, oxygen, nitrogen, hydrogen, and carbon atoms in AA molecules, respectively. Only half of the simulation box is shown here for clarity.

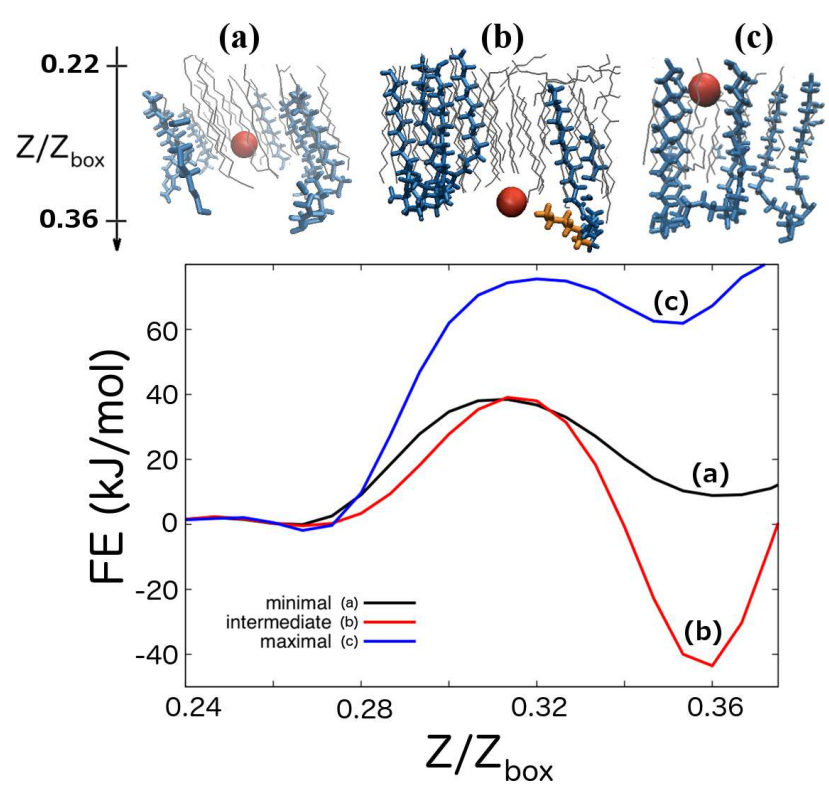

FIG. 3: Bottom: FEPs associated with three pathways obtained with the WT-metaD framework. Top: representative snapshots to illustrate the minimal (a), intermediate (b), and maximal (c) scenarios. Grey and blue lines represent $n$ dodecane and AA molecules, respectively. In the snapshots, the free methane molecule is shown as a red sphere. In (b), the AA short tail interacting with the methane molecule is colored orange. The $x$-axis corresponds to the $Z$-Cartesian coordinate of methane expressed in reduced units, $Z / Z_{\mathrm{box}}$, with $Z_{\text {box }}$ the size of the simulation box along the $Z$ direction.

travels through the interfacial layer and eventually interacts with the hydrophobic short tail of one AA, which can be parallel to the interfacial layer. This specific orientation enhances the interaction between the short tail and the methane molecule, stabilizing the system. This effect yields the inversion of the thermodynamic stability of the system with respect to the global $\left(Z / Z_{\text {box }} \approx 0.27\right)$ and local $\left(Z / Z_{\text {box }} \approx 0.36\right)$ minima observed along the minimal FEP. The maximal FEP corresponds to the diffusion of methane through a locally dense AA region, in which a few oil molecules are trapped within the AA layer (cf. Figure 3r). Possibly because the AA molecules are rigid in these simulations, the transport of methane across this region encounters a large FE barrier.

As discussed in the Supporting Information (SI), the FEP obtained within the metadynamics framework shows the same qualitative behavior as the FEP obtained within the ABMD/US framework, with quantitative differences caused by numerical artifacts. The remainder of the paper discusses the analysis on the minimal FE pathway, which shows the lowest FE barriers for transport of methane from the hydrocarbon to the hydrate (capture) and from the hydrate to the hydrocarbon (escape). In the following detailed analysis, we implemented the US and ABMD frameworks, as described previously. 
The minimal FEP would provide the lowest boundary for time constants representative for methane transport across the AAs film.

\section{Minimal free-energy pathway}

Thermodynamic properties

Two different systems were compared: 1) the AA layer is kept frozen, as in the case of the preliminary WTmetaD simulation and 2) the AA layer is flexible, although the central nitrogen atom of each AA molecule remains frozen. The former approach allows us to define unambiguously the Cartesian position of the free methane molecule with respect to the AA layer. Figure 4 compares the FEP associated to the minimal FE path, as obtained in the two approaches. In both cases, the AA molecules induce the alignment and rigidity of the oil molecules within the interface, as shown in Figure 2 and reported by Bui et al. [36]. Freezing the AA molecules does not impact the location of global and local minima, $Z / Z_{\text {box }}=0.27$ and $Z / Z_{\text {box }}=0.36$, respectively, and the $\mathrm{FE} \Delta F_{0} \approx 8.5 \mathrm{~kJ} / \mathrm{mol}$. This was expected as these quantities correspond to AA-free locations above and below the interfacial layer, which are not impacted by the AA freezing. However, freezing the AA molecules yields an increase of the FE barriers, $\Delta \Delta F$ $\equiv \Delta F_{c}^{\text {frozen }}-\Delta F_{c}^{\text {free }} \approx 7.5 \mathrm{~kJ} / \mathrm{mol}$ at the transition,

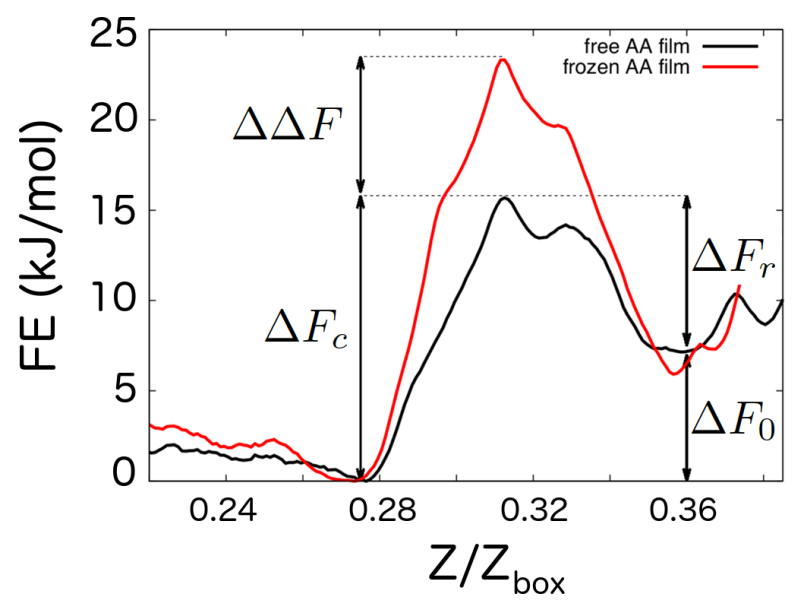

FIG. 4: FEPs associated with the passage of the free methane molecule across the interfacial layer, obtained within the US/ABMD framework. The AA surface density is $\approx 0.67$ molecules $/ \mathrm{nm}^{2}$. The $x$-axis corresponds to the $Z$-Cartesian coordinate of methane expressed in reduced units, $Z / Z_{\mathrm{box}}$, with $Z_{\text {box }}$ the size of the simulation box along the $Z$ direction. We show the minimal FE pathways obtained when the AA molecules are either frozen (red) or free (black). The activation energies associated with methane capture and escape, $\Delta F_{c}$ and $\Delta F_{r}$, are $\approx 15.5 \mathrm{~kJ} / \mathrm{mol}$ and $\approx 7 \mathrm{~kJ} / \mathrm{mol}$, respectively.
$Z / Z_{\text {box }} \approx 0.31$ (cf. Figure 4). This difference highlights the interplay between the rigidity of the AA layer and the local pliability of the oil molecules trapped within the interfacial layer.

\section{Transport Mechanisms}

Because the results obtained when the AA layer is flexible are more realistic, we further study the transport mechanism as observed within the US/ABMD framework. Figure 5 shows representative snapshots extracted from the FEP. The free methane molecule is initially in the bulk hydrocarbon phase, above the AA layer. When it comes closer to the interface, it is first trapped in a local $\mathrm{FE}$ minimum $\left(Z / Z_{\mathrm{box}} \approx 0.27\right)$. This minimum corresponds to a transition region between oil molecules isotropically oriented in the bulk and oil molecules parallel to the AA tails. The methane molecule then enters the AA layer between two hydrocarbon molecules. As the methane travels farther across the interfacial layer, an energy barrier arises as the oil molecules are displaced from the methane pathway. The transport proceeds until one oil molecule cannot be pushed farther down $\left(0.27 \lesssim Z / Z_{\text {box }} \lesssim 0.31\right)$. At these conditions, the oil molecule being displaced bends, eventually forming a cage surrounding the methane molecule $\left(Z / Z_{\mathrm{box}} \approx 0.31\right)$. This corresponds to the high energy transition region in the FE landscape. From this point on, the hydrocarbon forming the cage effectively contributes to actively pushing the methane towards the hydrate. The system evolves between two states corresponding to a closed or opened gate below the methane molecule $\left(0.31 \lesssim Z / Z_{\text {box }} \lesssim 0.34\right)$. Once the methane molecule overcomes this transition state, it is pushed down underneath the AA layer $\left(Z / Z_{\text {box }} \approx 0.35\right)$. The methane molecule then reaches the local minimum corresponding to the water layer between the AA layer and the hydrate. This analysis does not explore further transport through the water film.

\section{Dynamical Properties}

To estimate the transition rates along the $\mathrm{FE}$ pathway, we considered the Kramers theory 62 67], which is based on the inertial Langevin equation [68]:

$$
m \ddot{q}=-\frac{\partial V}{\partial q}-m \gamma \dot{q}+R(t) .
$$

In Eq. 1. $q$ represents the reaction coordinate, $m$ is the reduced mass for the reaction coordinate, $\gamma$ is the friction coefficient, and $V(q)$ is a PMF. $R(t)$ is a random force with zero mean that satisfies the fluctuation theorem [69]. In the presented case, Eq. 1 is applied to the methane 
(a)

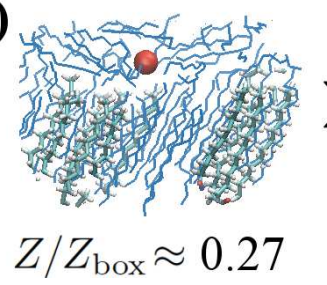

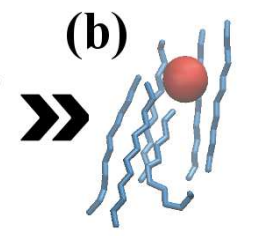

$Z / Z_{\mathrm{box}} \approx 0.29$

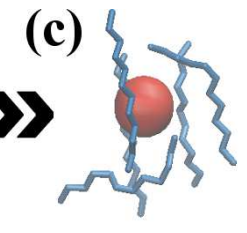

$Z / Z_{\mathrm{box}} \approx 0.31$

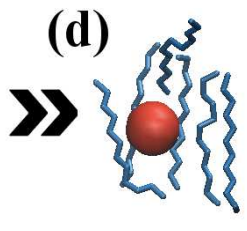

$Z / Z_{\mathrm{box}} \approx 0.32$

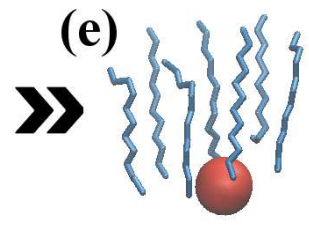

$Z / Z_{\mathrm{box}} \approx 0.36$

FIG. 5: Sequence of simulation snapshots representing the transport mechanism of the methane molecule (red sphere) along the minimal FE pathway across the flat interfacial layer composed of a mixture of AA and dodecane (blue molecules). The bulk hydrocarbon phase and the sII hydrate are not shown for clarity. The AA layer is only shown in (a). The methane molecule initially starts in the bulk hydrocarbon phase, above the AA layer (a). The methane molecule then enters the interfacial layer through two oil molecules (b). As the methane goes farther across the interfacial layer, the oil molecules below the methane molecule bend, eventually forming a cage surrounding the methane molecule (c). As the methane travels farther down, one oil molecule forming the gate begins pushing the methane molecule. At that stage, the system evolves between two states corresponding to a closed or opened gate below the methane molecule (d). Eventually, the methane molecule is driven underneath the AA layer (e).

molecule, the reaction coordinate is the $Z$-Cartesian coordinate, $m$ represents the methane molecule mass, and $V(q)$ is the PMF discussed in Figure 4. The escaping/capture rates can be described within the Kramers theory framework in terms of diffusion across a FE barrier of height $\Delta F$ when the barrier (and local minima) are modeled as parabolic potentials [66]:

$$
V(q)=V_{T S}-\frac{1}{2} m \omega_{T S}^{2}\left(q-q_{T S}\right)^{2} .
$$

The Kramers theory provides a physical derivation of the reaction rate constants in terms of the shape of the energy profile. From intermediate to high friction regimes, the reaction rate, $k$, is given as:

$$
k=\frac{\gamma}{\omega_{T S}}\left(\sqrt{\frac{1}{4}+\frac{\omega_{T S}^{2}}{\gamma^{2}}}-\frac{1}{2}\right) \frac{\omega_{0}}{2 \pi} e^{-\Delta F / k_{B} T}
$$

where $\omega_{0}$ and $\omega_{T S}$ represent the stiffness of the potential well and the barrier, respectively, $\gamma=6 \pi \eta R / m$ the friction coefficient, with $\eta$ the effective viscosity, and $\Delta F$ the FE barrier. Figure 6 shows the FEP fitted using harmonic potentials at the local minimum and maximum. This yields $\omega_{0} \approx 100 \times 10^{13} \mathrm{~s}^{-1}$ and $\omega_{T S} \approx 230 \times 10^{13} \mathrm{~s}^{-1}$ (cf. details in the SI). One important parameter is the effective viscosity $\eta$. Because of confinement of the liquid hydrocarbon at the interface, the effective viscosity is intrinsically different from its bulk value 61 ] $(\approx 2 \mathrm{mPa} . \mathrm{s}$ at $277 \mathrm{~K}$ and $20 \mathrm{MPa}$ ). Singer and Pollock [61] showed that the effective viscosity can increase by several orders of magnitude when dodecane molecules are confined in a space approaching the molecular dimension of the liquid hydrocarbon. Eventually, they observed the divergence of the effective viscosity. Considering $\eta \approx 10^{2}-10^{5}$ Pa.s [61], one obtains a lower boundary for the friction coefficient, $\gamma>15 \times 10^{18} s^{-1}>>\omega_{T S}$, characteristic of the strong friction regime of interest here. In this limit,
Eq. 3 simplifies to [66]

$$
k=\frac{\omega_{0} \omega_{T S}}{2 \pi \gamma} e^{-\Delta F / k_{B} T} .
$$

This approximation is often used to interpret, for instance, the time scale characteristic for protein or DNA conformational dynamics [70, 71]. Considering the parameter values discussed previously, one obtains a characteristic time scale for methane escape from the aqueous film near the hydrate to the hydrocarbon liquid phase, across the AA film, $\tau_{\text {esc }} \approx 0.01-1 \mu \mathrm{s}$.

Building on the recent method of Parrinello, Salvalaglio, and Tiwary [72, 73], we extend the metaD scope to assess numerically $\tau_{\text {esc }}$. We indicate our numerical result as $\tau_{\text {esc }}^{(\text {num })}$ in what follows. WT-metaD was performed using the $Z$ coordinate as $\mathrm{CV}$ with the parameters reported in the Methods section. The statistics for $\tau_{\text {esc }}^{\text {(num) }}$ conformed to a Poisson distribution with mean

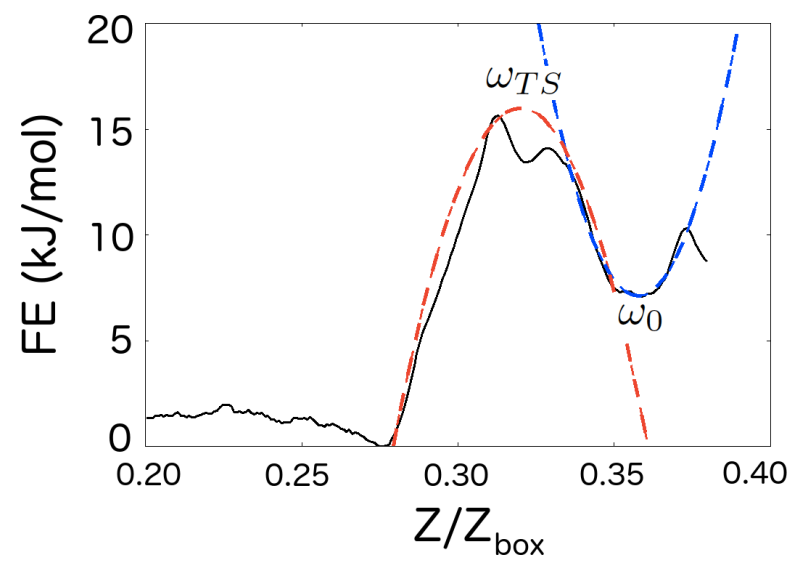

FIG. 6: Fitting of the FEP within parabolic potentials to extract parameters for the Kramers theory. The nonlinear leastsquares Marquardt-Levenberg algorithm was implemented obtaining $\omega_{0} \approx 100 \times 10^{13} \mathrm{~s}^{-1}$ and $\omega_{T S} \approx 230 \times 10^{13} \mathrm{~s}^{-1}$. 
$\mu=0.08 \pm 0.02 \mu \mathrm{s}$ and variance $\lambda=0.09 \mu \mathrm{s}$. The statistics obeys a two-sample KS test with $p$-value equal to 0.52 . This numerical result confirms the reliability of the choice of the $Z$-Cartesian coordinate as a collective variable in the US framework (cf. discussion in the SI) and is comparable to the estimation derived from the Kramers theory with a friction coefficient $\gamma \approx 10^{4} \mathrm{~Pa}$.s.

Finally we quantified the characteristic time scale for methane capture (from the hydrocarbon to the hydrate surface) along the minimal FE path, $\tau_{\text {cap }}$. As shown in Figure 6. no confinement was present above the AA layer $\left(Z / Z_{\text {box }}<0.27\right)$. Thus the entropic effect would dominate, and the associated rate of capture would tend to infinity. To correct for this, an external potential was added at $Z / Z_{\mathrm{box}} \approx 0.25$, similar to the $\mathrm{FE}$ barrier observed for $Z / Z_{\text {box }} \in[0.27,0.31]$. This external potential would represent, for instance, a free methane molecule confined between two AA layers. The statistics of our numerical analysis for $\tau_{\text {cap }}^{(\text {num })}$ conformed to a Poisson distribution with mean $\mu=0.35 \pm 0.15 \mu$ s and variance $\lambda=0.49 \mu \mathrm{s}$, and followed a two-sample KS test with $p$-value equal to 0.51 (cf details in the SI).

\section{Intermediate free-energy pathway}

To conclude this analysis, we considered the intermediate path defined in Figure 3, which presents a similar FE barrier associated with methane capture but a deeper energy basin at $Z / Z_{\text {box }} \approx 0.35$ along with a higher FE barrier associated with methane escape. We reconstructed the accurate FEP within the US/ABMD framework and obtained the respective values for $\omega_{T S} \approx 100 \times 10^{13} \mathrm{~s}^{-1}$ and $\omega_{0} \approx 130 \times 10^{13} \mathrm{~s}^{-1}$. The details are reported in the SI. Considering Eq. 4 with $\gamma \approx 10^{4}$ Pa.s, we obtained a characteristic time scale for methane escape, $\tau_{\text {esc }} \approx 40 \mu \mathrm{s}$. The estimated characteristic time scale for methane capture was similar to that obtained in correspondence of the minimal path.

\section{Discussion}

The extensive simulations discussed previously allowed for identification of three possible pathways that a methane molecule can follow to diffuse from the hydrocarbon phase to the hydrate structure across the interfacial film rich in AAs. We focused on the pathway that showed the minimal FE barrier and we quantified accurately the FEPs using a combination of enhanced sampling techniques. This allowed us to reveal the molecular mechanism responsible for the $\mathrm{FE}$ barrier. Interpreting the FEP within the Kramers theory allowed us to extract the time constant that quantifies the escape rate of the methane across the AA film. The result is consistent with estimations from a direct metaD estimate, which supports the reliability of our approach.

We showed that the FE barrier comes from local flexibility of the liquid hydrocarbon molecules within the AA film. The flexibility of the oil molecules is impacted by the rigidity of the AA molecules. We observed that the interaction of the methane molecule with the AA short tail can increase the stability of the system and invert its thermodynamic stability. Increasing the AA surface density leads to an increase in the heigh of the FE barrier, improving eventually the efficiency of the interfacial layer in limiting the transport of methane.

The characteristic time obtained from this study can be compared with the experimental growth rate of methane hydrate film at flat methane/water interface. Sun et al. 74, and more recently Li et al. 25] reported growth characteristic time $\tau^{\exp } \approx 1-5 \mu \mathrm{s}$, depending on the degree of supercooling considered in the experiments. This is one order of magnitude higher than the escaping time obtained in our simulations for the minimal FE pathway and one order of magnitude lower than that obtained for the intermediate FE pathway. These data suggest that ordering of the long AA tails observed for the AAs considered here can provide effective barriers to methane transport. Further, the results suggest that the short tails of the AAs can be designed to enhance the stability of methane near the growing hydrate.

The new physical insights discussed in this paper could be useful for a variety of applications. For instance, several research groups focus on the interplay between design and performance of AAs at the microscopic scale. Accounting for the collective effect of new AAs could allow us to infer their use in practical applications, which addresses the current needs.

\section{METHODS}

\section{Unbiased MD simulations}

MD simulations were performed with the GROMACS software package, version 5.1.1 56] using the TIP4P/Ice water model [75]. Biased simulations were performed using version 2.3 of the plugin for $\mathrm{FE}$ calculation, PLUMED [76]. The TIP4P/Ice model has been successfully implemented to study hydrate nucleation and growth [4, 77] and to investigate the performance of potential hydrate inhibitors [78]. This model yields an equilibrium temperature for the formation of gas hydrates at high pressure close to experimental values [79]. Methane and $n$-dodecane were represented within the united-atom version of the TraPPE-UA force field [80]. AAs were modeled using the general Amber force field (GAFF) [81], which is often implemented for modeling organic and pharmaceutical molecules containing $\mathrm{H}, \mathrm{C}, \mathrm{N}, \mathrm{O}, \mathrm{S}, \mathrm{P}$, and halogens. Atomic charges were calculated with the 
AM1-BCC method employed in Antechamber from the Amber 14 suite 82]. The chloride counterions $\left(\mathrm{Cl}^{-}\right)$were modeled as charged Lennard-Jones (LJ) spheres with the potential parameters taken from Dang [83], without polarizability. The sII hydrates were considered to be the solid substrate, and they were not allowed to vibrate in this work. AAs, chloride counterions, $n$-dodecane, and methane composed the liquid phase. Dispersive and electrostatic interactions were modeled by the $12-6$ LJ and Coulombic potentials, respectively. The LorentzBerthelot mixing rules [84, 85] were applied to determine the LJ parameters for unlike interactions from the parameters of the pure components. The distance cutoff for all non-bonded interactions was set to $1.4 \mathrm{~nm}$. Long-range corrections to the electrostatic interactions were described using the particle mesh Ewald (PME) method 86 88] with a Fourier grid spacing of $0.12 \mathrm{~nm}$, a tolerance of $10^{-5}$, and fourth-order interpolation. Periodic boundary conditions were applied in three dimensions for all simulations.

To construct the initial configurations, we followed the procedure described by Bui et al. [36]. One unit cell of sII methane hydrates was adapted from the study of Takeuchi et al. [89]. The sII methane hydrate unit cell was replicated three times in the $X$ and $Y$ directions $(5.193 \mathrm{~nm})$ and two times in the $Z$ direction $(3.462 \mathrm{~nm})$. It was then flanked by a thin liquid water film of approximately $0.5 \mathrm{~nm}$ on both sides along the $\mathrm{Z}$ direction. The desired number of AA molecules was arranged near both sides of the hydrate substrate. The chloride counterions $\left(\mathrm{Cl}^{-}\right)$were placed next to the AA headgroups. The $n$ dodecane and methane molecules were placed within the remainder of the simulation box. The time step used in all the simulations was $0.001 \mathrm{ps}$, and the list of neighbors was updated every $0.01 \mathrm{ps}$ with the grid method and a cutoff radius of $1.4 \mathrm{~nm}$.

The energy of the model was first optimized with the "steepest descent minimization" algorithm to remove high-energy configurations. Subsequently, to minimize the possibility that the initial configuration biased the simulation results, an NVT temperature-annealing procedure, as implemented in GROMACS [56], was conducted. The algorithm linearly decreased the system temperature from $1000 \mathrm{~K}$ to $277 \mathrm{~K}$ in $500 \mathrm{ps.} \mathrm{In} \mathrm{these}$ simulations, the hydrate substrate and chloride ions were kept frozen. To relax the structure of $n$-dodecane and AAs, a $N V T$ simulation was conducted at $277 \mathrm{~K}$ for 2 ns using the Berendsen thermostat [90], with the sII hydrate structure kept frozen. The equilibration phase was then conducted within the isobaric-isothermal (NPT) ensemble under thermodynamic conditions favorable for hydrate formation $(T=277 \mathrm{~K}$ and $P=20 \mathrm{MPa})$ to equilibrate the density. During the NPT simulation, all molecules in the system were allowed to move, including water and methane molecules in the hydrate substrate. The pressure coupling was applied only along the $Z$ di- rection of the simulation box, which allowed the $X$ and $Y$ dimensions to be maintained constant. Temperature and pressure were maintained at $277 \mathrm{~K}$ and $20 \mathrm{MPa}$, respectively using the Berendsen thermostat and barostat 90 for $5 \mathrm{~ns}$. This is considered the most efficient algorithm to scale simulation boxes at the beginning of a simulation 91. We then switched to the Nose-Hoover thermostat [92] and the Parrinello-Rahman barostat [93] for 100 ns, which are considered more thermodynamically consistent algorithms 91]. This numerical protocol allowed the AAs to assemble and orient to form the ordered layer described in the work of Bui et al. [36]. The system was then equilibrated for $3 \mathrm{~ns}$ in $N V T$ conditions coupling with the $v$-rescale thermostat 94 $\left(T=277 \mathrm{~K}, \tau_{T}=0.1\right.$ $\mathrm{ps})$. To define the position of the free methane molecule in the simulation box with respect to the sII hydrate structure, the simulation was continued in $N V T$ conditions holding in place the methane molecule enclathrated into the water cages defining the sII hydrate structure, as implemented with the freeze group procedure in GROMACS [56].

\section{Biased MD simulations}

The phenomenon of interest (i.e. the transport of methane across the interfacial layer) occurs on time scales that are orders of magnitude longer than the accessible time that can be currently simulated with classical MD simulations. A variety of methods, referred to as enhanced sampling techniques [95 99], can be implemented to overcome this limitation. These methods accelerate rare events and are based on constrained MD. MetaD [54, 100 102] and US [55, 103] belong to this class of methods: they enhance the sampling of the conformational space of a system along a few selected degrees of freedom, named reaction coordinates or collective variables $(\mathrm{CVs})$, and reconstruct the probability distribution as a function of these CVs. These techniques are proven powerful tools to study biological 71, 104 106. and chemical systems $107-109]$. However, despite these successes, care should be taken to properly choose and implement the reaction coordinates 100 102].

We first ran a well-tempered metaD (WT-metaD) simulation [110, 111] using the three Cartesian coordinates $(X, Y$ and $Z$ ) of the free methane molecule as CVs. WTmetaD is a method based on a biasing of the potential surface. The biasing potential is dynamically placed on top of the underlying potential energy landscape to discourage the system from visiting the same points in the configurational space. The WT-metaD time-dependent bias, $V_{\text {bias }}(s, t)$ can have any form, but a Gaussian po- 
tential is usually implemented [100]:

$$
V_{\text {bias }}(s, t)=\omega \sum_{t^{\prime}<t} \exp \left[-\frac{\left(s(t)-s\left(t^{\prime}\right)\right)^{2}}{2 \sigma^{2}}\right] .
$$

In Eq. $5 \omega$ is the height of the biasing potential, $\sigma$ is the width, $t$ is the time, and $s$ is the collective variable. Following the algorithm introduced by Barducci et al. [110], a Gaussian-shaped potential is deposited every $\tau_{G}=2 \mathrm{ps}$, with height $\omega=\omega_{0} e^{-V(s, t) /(f-1) T}$, where $\omega_{0}=5 \mathrm{~kJ} / \mathrm{mol}$ is the initial height, $T$ is the temperature of the simulation, and $f \equiv(T+\Delta T) / T=25$ is the bias factor with $\Delta T$ a parameter with the dimension of a temperature.

In our implementation, the resolution of the recovered FE surface is determined by the width of the Gaussian $\sigma=0.25 \mathrm{~nm}$ along the $X, Y$ and $Z$ directions. This step was motivated by the inherent competition between different pathways that can lead to methane transport through the AA layer, with each path characterized by different FE barriers. WT-metaD simulations were run restraining the position of the AA layer while allowing the hydrocarbon molecules, both trapped at the interface and present in the bulk phase, to move freely. This was necessary to minimize local hysteresis effects that would occur in the global convergence of the WT-metaD resulting from the flexibility of the AA layer in defining the Cartesian position of the free methane molecule with respect to the AA film.

Once the possible pathways across the AA film were identified, the potential of mean force (PMF) along them was rigorously calculated using US. This combined metaD/US approach, originally proposed by Zhang and Voth [112] in the biophysical context, provides a powerful means to calculate a physically meaningful PMF where the convergence problem, sometimes associated with metaD, is avoided. The approach also avoids the discontinuity problem, often associated with US calculations that assume a straight-line reaction coordinate, yielding a physically accurate PMF [112]. It is worth noting that in the combined metaD/US approach, the final PMF is not sensitive to the choice of the WT-metaD parameters, as long as they are in reasonable range because the PMF is not directly calculated from metaD 112]. In the WT-metaD simulations, soft walls were added on both side of the interfacial layer to limit sampling inside the AA film.

Once representative pathways were identified, we calculated the associated PMF using US, with the $Z-$ Cartesian coordinate along the preliminary pathways as collective variable. The system was free in the $X-Y$ plane. To design the US windows, it is common practice to implement one of two approaches: 1) the system is dragged using the steered dynamics 103 or 2) the initial structures are prepared by changing the position of the CV directly followed by energy minimization [113]. As discussed by Nishizawa [113], it is possible, with these protocols, to drive the system into configurations with no reverse transition (i.e., breaking the ergodicity of the system). This can be particularly true with fluid/soft systems, where covalent bonds are not present. This shortcoming can be associated to artifactual kinetic barriers between consecutive states that slow sampling of configurations during US. These effects could render the free-energy profile (FEP) analysis inappropriate and/or unfeasible using a reasonable amount of computation. To avoid these shortcomings, we used the adiabatic biased molecular dynamics (ABMD) framework, which is based on the local fluctuations of the system [114 117] .

Furthermore, one must avoid inefficient sampling in the CV orthogonal degrees of freedom (either $X-$ or $Y$ - direction), despite ensuring good overlap in the $Z-$ direction. As others indicated [112, 118, 119], this numerical issue could cause a discontinuity in configurational space that would introduce PMF errors. To ensure the results are reliable, we controlled the efficiency of the sampling along the CV orthogonal degrees of freedom with the implementation of the flat-bottomed potential 112, 118, 119.

$$
V_{f b}(q)=\frac{1}{2} k_{f b} \Theta\left[\rho(q)-R_{f b}\right]\left(\rho(q)-R_{f b}\right)^{2},
$$

where $q$ denotes the $X$ - or $Y$ - direction, $\rho(q)$ the distance from the path along which the US windows are distributed, $k_{f b}$ the harmonic force constant, $\Theta(q) \equiv$ $1-\Pi(q)$ with $\Pi(q)$ the rectangular function, and $R_{f b}$ the confinement radius. No constraint was added in the $X-Y$ plane in the present PMF calculations using the $Z$-coordinate as $\mathrm{CV}$, unless the methane molecule is close to the bulk solvent or the hydrate. Therefore, as $V_{f b}(q)$ is flat-bottomed, it has no effect when the methane molecule travels through the interfacial layer. This bias only affects the overall offset of the reconstructed FEP in the layer and not its shape [119].

Upon completion of the US simulations, FEPs were calculated from the final 4 ns of simulation time using the weighted histogram analysis method (WHAM) [120]. Statistical error analysis was conducted using the integrated Monte Carlo bootstrapping framework [? ], implemented in WHAM [120], as discussed in the SI.

To estimate the rate of the methane transport across the interfacial layer, we extended the standard scope of WT-metaD considering the recent method or Parrinello, Salvalaglio and Tiwary [72, 73]. This technique has been applied to study transition rates in biological and chemical systems [58, 71, 121 125]. We denote by $\tau$ the physical mean transition time for the methane to pass over the energy barrier and by $\tau_{M}$ the mean transition time obtained from the WT-metaD run. The latter is linked to the physical mean transition time, $\tau$, by means of the 
acceleration factor,

$$
\alpha(t)=\tau / \tau_{M}=\left\langle e^{\beta V_{\text {bias }}(s, t)}\right\rangle_{M},
$$

where the angular brakets denote an average over a WTmetaD run confined to a metastable basin, and $V_{\text {bias }}(s, t)$ is the WT-metaD time-dependent bias defined in Eq. 5 . To avoid depositing bias in the transition state region, we increase the time lag between two successive Gaussian depositions in the WT-metaD framework, $\tau_{G}=400$ ps [2, 73]. We confirmed that the statistics of transition times follows a Poisson distribution, performing a two-sample Kolmogorov-Smirnov (KS) test. The compliance to the KS test allowed us to asses the reliability of the CV considered to distinguish between the different metastable states within the US framework [72, 73] and to reconstruct accurately the PMFs.

\section{Acknowledgements}

The authors thank Matteo Salvalaglio for fruitful discussion concerning the metaD algorithm. This work was granted to the HPC resources of the ARCHER UK National Supercomputing Service (http://www.archer.ac.uk). The authors are grateful for the financial support provided by Halliburton and the UK Engineering and Physical Sciences Research Council (EPSRC) under grant numbers 527889 and $\mathrm{EP} / \mathrm{N} 007123 / 1$.

* Corresponding author: francois.sicard@free.fr.

[1] C. Koh, R. Westacott, W. Zhang, K. Hirachand, J. Creek, and A. Soper, Fluid Phase Equilib. 194-197, 143 (2002).

[2] M. Kelland, Energy Fuels 20, 825 (2006).

[3] E. Sloan and C. Clathrate hydrates of natural gases, 3rd Ed $\quad$ Koh, Press: Boca Raton, Florida, 2008).

[4] M. Walsh, C. Koh, E. Sloan, A. Sum, and D. Wu, Science 326, 1095 (2009).

[5] V. Michalis, J. Costandy, I. Tsimpanogiannis, A. Stubos, and I. Economou, J. Chem. Phys. 142, 044501 (2015).

[6] P. Brewer, F. O. Jr., G. Friederich, K. Kvenvolden, D. Orange, J. McFarlane, and W. Kirkwood, Geology 25, 407 (1997).

[7] A. Sum, C. Koh, and E. Sloan, Ind. Eng. Chem. Res. 48, 7457 (2009).

[8] K. Kaiho, T. Arinobu, R. Ishiwatari, H. Morgans, H. Okada, N. Takeda, K. Tazaki, G. Zhou, Y. Kajiwara, R. Matsumoto, et al., Paleoceanography 11, 447 (1996).

[9] K. Park, Z. Ni, A. Côté, J. Choi, R. Huang, F. UribeRomo, H. Chae, M. OKeeffe, and O. Yaghi, Proc. Natl. Acad. Sci. U.S.A. 103, 12690 (2006).

[10] E. Sloan, Nature 426, 353 (2003).

[11] L. Florusse, C. Peters, J. Schoonman, K. Hester, C. Koh, S. Dec, K. Marsh, and E. Sloan, Science 306, 469 (2004).
[12] W. Mao, H. Mao, A. Goncharov, V. Struzhkin, Q. Guo, J. Hu, J. Shu, R. Hemley, M. Somayazulu, and Y. Zhao, Science 297, 2247 (2002).

[13] T. Ogawa, T. Ito, K. Watanabe, K. ichi Tahara, R. Hiraoka, J. ichi Ochiai, R. Ohmura, and Y. Mori, Appl. Therm. Eng. 26, 2157 (2006).

[14] C. Fisher, I. MacDonald, R. Sassen, C. Young, S. Macko, S. Hourdez, R. Carney, S. Joye, and E. McMullin, Naturwissenschaften 87, 184 (2000).

[15] D. Milton, Science 183, 654 (1974).

[16] E. Sloan, C. Koh, A. Sum, A. Ballard, J. Creek, M. Eaton, J. Lachance, N. McMullen, T. Palermo, G. Shoup, et al., Natural Gas Hydrates in Flow Assurance, 3rd Ed. (Gulf Professional Pub./Elsevier:Burlington, Massachusetts, 2011).

[17] J. Mullin, Chapter 5: Nucleation. In Crystallization, 4th Ed. (Butterworth-Heinemann: Oxford (UK), 2011).

[18] E. Freer, M. Selim, and E. Sloan, Fluid Phase Equilib. 185, 65 (2001).

[19] D. Turner, Clathrate Hydrate Formation in Water-in-oil Dispersions (Ph.D. Thesis, Colorado School of Mines, Golden (CO), April 2005).

[20] E. Sloan, Hydrate Engineering (Society of Petroleum Engineers, Richardson (TX), 2000).

[21] J. Lee, S. Cho, J. Lee, P. Linga, K. Kang, and J. Lee, Energy Technol. 3, 925 (2015).

[22] O. Fandino and L. Ruffine, Fuel 117, 442 (2014).

[23] S. Kulkarni, S. Kadam, H. Meekes, A. Stankiewicz, and J. ter Horst, Cryst. Growth Des. 13, 2435 (2013).

[24] H. Abay and T. Svartaas, Energy Fuels 25, 42 (2011).

[25] S. Li, C. Sun, B. Liu, Z. Li, G. Chen, and A. Sum, Sci. Rep. 4, 1 (2014).

[26] W. Ke and M. Kelland, Energy Fuels 30, 10015 (2016).

[27] D. Yuhara, B. Barnes, D. Suh, B. Knott, G. Beckham, K. Yasuoka, D. Wu, and A. Sum, Faraday Discuss. 179, 463 (2015).

[28] C. Moon, R. Hawtin, and P. Rodger, Faraday Discuss. 136, 367 (2007).

[29] J. Vatamanu and P. Kusalik, J. Phys. Chem. B 26, 15896 (2006).

[30] A. Sum, C. Koh, and E. Sloan, Energy Fuels 26, 4046 (2012).

[31] C. R. Jr. and P. Large, Chem. Eng. Sci. 63, 2007 (2008).

[32] L. Zhang, C. Zhang, H. Huang, D. QI, Y. Zhang, S. Ren, Z. Wu, and M. Fang, Petrol. Explor. Develop. 41, 824 (2014).

[33] J. Lederhos, J. Long, A. Sum, R. Christiansen, and E. Sloan, Chem. Eng. Sci. 51, 1221 (1996).

[34] H. Zhao, M. Sun, and A.Firoozabadi, Fuel 180, 187 (2016).

[35] E. Sloan, U.S. Patent US5880319 A (May 25, 1994).

[36] T. Bui, A. Phan, D. Monteiro, Q. Lan, M. Ceglio, E. Acosta, P. Krishnamurthy, and A. Striolo, Langmuir 33, 2263 (2017).

[37] M. Kelland, T. Svartaas, and L. Andersen, J. Pet. Sci. Eng. 64, 1 (2009).

[38] Z. Aman, E. Sloan, A. Sum, and C. Koh, Phys. Chem. Chem. Phys. 16, 25121 (2014).

[39] M. mechanisms responsible for hydrate anti-agglomerant performance, Phys. Chem. Chem. Phys. 18, 24859 (2016).

[40] T. Bui, F. Sicard, D. Monteiro, Q. Lan, M. Ceglio, C. Burress, and A. Striolo, Nano Lett., submitted for 
publication (2018).

[41] M. Zanota, C. Dicharry, and A. Graciaa, Energy Fuels 19, 584 (2005).

[42] J. Chen, C. Sun, B. Peng, B. Liu, S. Si, and M. J. et al., Energy Fuels 27, 2488 (2013).

[43] M. Kelland, T. Svartaas, J. Ovsthus, T. Tomita, and J. Chosa, Chem. Eng. Sci. 61, 4048 (2006).

[44] M. Sun, Y. Wang, and A. Firoozabadi, Energy Fuels 26, 5626 (2012).

[45] J. Chen, J. Liu, C. Sun, M. Jia, and B. L. et al., Energy Convers. Manage. 86, 886 (2014).

[46] J. Chen, Y. Wang, C. Sun, F. Li, N. Ren, M. Jia, K. Yan, Y.-N. Lv, B. Liu, and G.-J. Chen, Energy Fuels 29, 122 (2015).

[47] S. Gao, Energy Fuels 23, 2118 (2009).

48] M. Kelland, Production Chemicals fo (CRC Press: Boca Raton, Florida, 2014).

[49] M. Lauricella, S. Meloni, N. English, B. Peters, and G. Ciccotti, J. Phys. Chem. C 118, 22847 (2014).

[50] M. Lauricella, S. Meloni, S. Liang, N. English, P. Kusalik, and G. Ciccotti, J. Chem. Phys. 142, 244503 (2015).

[51] K. Hall, S. Carpendale, and P. Kusalik, Proc. Nat. Acad. Soc. U.S.A. 113, 12041 (2016).

[52] F. Jimenez-Angeles and A. Firoozabadi, Proceeding of the 9th International Conference on Gas Hydrates, Denver, Colorado, USA (June 25-30, 2017).

[53] M. Bellucci, M. Walsh, and B. Trout, Proceeding of the 9th International Conference on Gas Hydrates, Denver, Colorado, USA (June 25-30, 2017).

[54] A. Laio and M. Parrinello, Proc. Nat. Acad. Soc. U.S.A. 99, 1256212566 (2002).

[55] G. Torrie and J.P.Valleau, J. Comput. Phys. 23, 187 (1977).

[56] M. Abraham, T. Murtola, R. Schulz, S. Páll, J. Smith, B. Hess, and E. Lindahl, SoftwareX 1-2, 19 (2015).

[57] V. Limongelli, L. Marinelli, S. Cosconati, C. L. Motta, S. Sartini, L. Mugnaini, F. D. Settimo, E. Novellino, and M. Parrinello, Proc. Natl. Acad. Sci. U.S.A. 109, 1467 (2012).

[58] P. Tiwary, J. Mondal, J. Morrone, and B. Berne, Proc. Nat. Acad. Soc. U.S.A. 112, 12015 (2015).

[59] J. Yang, M. Bonomi, C. Calero, and J. Mart, Phys. Chem. Chem. Phys. 18, 9036 (2016).

[60] S. Bhakat and P. Soderhjelm, J. Comput. Aided Mol. Des. 31, 119 (2017).

[61] S. Granick, Fundamentals of Friction, Macroscopic and Micr (I.L. Singer and H. Pollock Eds.; Springer Netherlands, 1992).

[62] H. Kramers, Physica (Amsterdam) 7, 284 (1940).

[63] P. Hanggi, P. Talkner, and M. Borkovec, Rev. Mod. Phys. 82, 251 (1990).

[64] E. Pollak and P. Talkner, Chaos 15, 026116 (2005).

[65] H.-X. Zhou, Quaterly Rev.Biophys. 43, 219 (2010).

[66] B. Peters, Reaction Rate Theory and Rare Events, 1st Ed. (Elsevier: Amsterdam, The Netherlands, 2017).

[67] F. Sicard, arXiv:1803.03490 [cond-mat.stat-mech] (2018).

[68] W. Coffey and Y. Kalmykov, The Langevin Equation: With Applications to Stochastic Pr vol. 27 (World Scientific Publishing Company: Singapore, 2012).

[69] U. Marini, B. Marconi, A. P. L. Rondoni, and A. Vulpiani, Phys. Rep. 461, 111 (2008).

[70] H. Yu, A. Gupta, X. Liu, K. Neupane, A. Brigley, I. Sosova, and M. Woodside, Proc. Nat. Acad. Sci. U.S.A.
109, $14452(2012)$

[71] F. Sicard, N. Destainville, and M. Manghi, J. Chem. Phys. 142, 034903 (2015).

[72] P. Tiwary and M. Parrinello, Phys. Rev. Lett. 111, 230602 (2013).

[73] M. Salvalaglio, P. Tiwary, and M. Parrinello, J. Chem. Theory Comput. 10, 1420 (2014).

[74] C. Sun, B. Peng, A. Dandekar, Q. Ma, and G. Chen, Annu. Rep. Prog. Chem., Sect. C 106, 77 (2010).

[75] J. Abascal, E. Sanz, R. F. Garcia, and C. Vega, J. Chem. Phys. 122, 234511 (2005).

[76] G. Tribello, M. Bonomi, D. Branduardi, C. Camilloni, and G. Bussi, Comput. Phys. Comm. 185, 604 (2014).

[77] L. Jensen, K. Thomsen, N. von Solms, S. Wierzchowski, M. Walsh, C. Koh, E. Sloan, D. Wu, and A. Sum, J. PHysdChem. B 114, 5775 (2010).

[78] S. A. Bagherzadeh, S. Alavi, J. Ripmeester, and P. Englezos, Phys. Chem. Chem. Phys. 17, 9984 (2015).

[79] M. Conde and C. Vega, J. Chem. Phys. 133, 064507 (2010).

[80] M. Martin and J. Siepmann, J. Phys. Chem. B 102, 2569 (1998).

[81] J. Wang, R. Wolf, J. Caldwell, P. Kollman, and D. Case, J. Comput. Chem. 25, 1157 (2004).

[82] D. Case, J. Berryman, R. betz, Q. Cai, D. Cerutti, T. Cheatham, T. Darden, R. Duke, H. Gohlke, A. Goetz, et al., AMBER 14 (University of California: San Francisco, California, 2014).

[83] D. Smith and L. Dang, J. Chem. Phys. 100, 3757 (1994).

[84] H. Lorentz, Ann. Phys. 248, 127 (1881).

[85] D. Berthelot, Compt. Rendus 126, 1703 (1898).

[86] T. Darden, D. York, and L. Pedersen, J. Chem. Phys. 98, 10089 (1993).

[87] U. Essmann, L. Perera, and M. Berkowitz, J. Chem. Phys. 103, 8577 (1995).

[88] M. Kawata and U. Nagashima, Chem. Phys. Lett. 340, 165 (2001).

[89] F. Takeuchi, M. Hiratsuka, R. Ohmura, S. Alavi, A. Sum, and K. Yasuoka, J. Chem. Phys. 138, 124504 (2013).

[90] H. Berendsen, J. Postma, W. van Gunsteren, A. DiNola, and J. R. Haak, J. Chem. Phys. 81, 3684 (1984).

[91] S. Pronk, S.Páll, R. Schulz, P. Larsson, P. Bjelkmar, R. Apostolov, M. Shirts, J. Smith, P. Kasson, D. van der Spoel, et al., Bioinformatics 29, 845 (2013).

[92] D. Evans and B. Holian, J. Chem. Phys. 83, 4069 (1985).

crdis3pid. Hironisselo 3andEA. Rahman, J. Appl. Phys. 52, 7182 (1981).

[94] G. Bussi, D. Donadio, and M. Parrinello, J. Chem. Phys. 126, 014101 (2007).

[95] S. A. J. McCammon, Chem. Rev. 106, 1589 (2006).

[96] V. Spiwok, Z. Sucur, and P. Hosek, Biotechnology Adv. 33, 1130 (2015).

[97] R. Bernardi, M. Melo, and K. Schulten, Biochim. Biophys. Acta. 1850, 872 (2015).

[98] T. Maximova, R. Moffatt, R. Nussinov, and A. Shehu, PLoS Comput. Biol. 12, e1004619 (2016).

[99] F. Pietrucci, Rev. Phys. 2, 32 (2017).

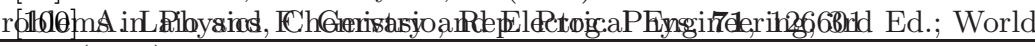
(2008).

[101] A. Barducci, M. Bonomi, and M. Parrinello, WIREs Comput. Mol. Sci. 1, 826 (2011).

[102] L. Sutto, S. Marsili, and F. Gervasio, WIREs Comput. Mol. Sci. 2, 771 (2012).

[103] J. Kastner, WIREs Comput. Mol. Sci. 1, 932 (2011). 
[104] A. Barducci, R. Chelli, P. Procacci, V. Schettino, F. Gervasio, and M. Parrinello, J. Am. Chem. Soc. 128, 2705 (2006).

[105] L. Sutto and F. Gervasio, Proc. Nat. Acad. Soc. 110, 10616 (2013).

[106] F. Sicard and P. Senet, J. Chem. Phys. 138, 235101 (2013).

[107] F. Giberti, M. Salvalaglio, and M. Parrinello, IUCrJ 2, 256 (2015).

[108] M. Salvalaglio, M. Mazzotti, and M. Parrinello, Faraday Discus. 179, 291 (2015).

[109] I. Gimondi and M. Salvalaglio, J. Chem. Phys. 147, 114502 (2017).

[110] A. Barducci, G. Bussi, and M. Parrinello, Phys. Rev. Lett. 100, 020603 (2008).

[111] J. Dama, M. Parrinello, and G. Voth, Phys. Rev. Lett. 112, 240602 (2014).

[112] Y. Zhang and G. Voth, J. Chem. Theory Comput. 7, 2277 (2011).

[113] M. Nishizawa and K. Nishizawa, Biophys. J. 104, 1038 (2013).

[114] E. Paci and M. Karplus, J. Mol. Biol. 288, 441 (1999).

[115] M. Marchi and P. Ballone, J. Chem. Phys. 110, 3697
(1999).

[116] C. Camilloni, R. Broglia, and G. Tiana, J. Chem. Phys. 134, 045105 (2011).

[117] F. Sicard and A. Striolo, Faraday Discuss. 191, 287 (2016).

[118] T. Allen, O. Andersen, and B. Roux, Proc. Nat. Acad. Sci. U.S.A. 101, 117 (2004).

[119] F. Zhu and G. Hummer, J. Chem. Theory Comput. 8, 3759 (2012).

[120] A. Grossfiled, http://membrane.urmc.rochester.edu/content/wham (2013).

[121] J. Schneider and K. Reuter, J. Phys. Chem. Lett. 5, 3859 (2014).

[122] M. Salvalaglio, C. Perego, F.Giberti, M. Mazzotti, and M. Parrinello, Proc. Nat. Acad. Sci. U.S.A. 112, E6 (2015).

[123] P. Tiwary, V. Limongelli, M. Salvalaglio, and M. Parrinello, Proc. Nat. Acad. Sci. U.S.A. 112, E386 (2015).

[124] P. Piaggi, O. Valssonbc, and M. Parrinello, Faraday Discuss. 195, 557 (2016).

[125] D. Bochicchio, M. Salvalaglio, and G. Pavan, Nat. Commun. 8, 147 (2017).

\section{Emergent Properties of Antiagglomerant Films Control Methane Transport: Implications for Hydrate Management}

\section{Supporting Information}

\section{IDENTIFICATION OF REPRESENTATIVE PATHWAYS}

Metadynamics simulations. The well-tempered metadynamics (WT-metaD) framework [S1, S2] was implemented to identify representative pathways available to the methane molecule to cross the antiagglomerants (AAs) film. We used the three Cartesian coordinates $(X, Y$ and $Z)$ of the free methane molecule as collective variables $(\mathrm{CVs})$. A Gaussian potential was used as time-dependent bias, $V_{\text {bias }}(s, t)[\mathrm{S} 3]$ :

$$
V_{\mathrm{bias}}(s, t)=\omega \sum_{t^{\prime}<t} \exp \left[-\frac{\left(s(t)-s\left(t^{\prime}\right)\right)^{2}}{2 \sigma^{2}}\right]
$$

In Eq. S1, $\omega$ is the height of the biasing potential, $\sigma$ is the width, $t$ is the time, and $s$ is the collective variable. Following the algorithm introduced by Barducci et al. [S1], a Gaussian-shaped potential was deposited every $\tau_{G}=2$ ps, with height $\omega=\omega_{0} e^{-V(s, t) /(f-1) T}$, where $\omega_{0}=5 \mathrm{~kJ} / \mathrm{mol}$ is the initial height, $T=277 \mathrm{~K}$ is the temperature of the simulation, and $f \equiv(T+\Delta T) / T=25$ is the bias factor with $\Delta T$ a parameter with the dimension of a temperature. In the implementation, the resolution of the recovered free-energy $(\mathrm{FE})$ surface is determined by the width of the Gaussian $\sigma=0.25 \mathrm{~nm}$ along the $X, Y$ and $Z$ directions. WT-metaD simulations were run restraining the position of the AA layer, while allowing the hydrocarbon molecules, both at the interface and in the bulk phase, to move freely. Soft walls were added on both sides of the interfacial layer to limit sampling inside the AA film.

Figure S1 shows the temporal evolution of the FEP reconstructed along the minimal FE path, from the $170 \mathrm{~ns}$ WT-metaD simulation. The FEP reached local convergence after 20 ns. However, after 64 ns the FEP began to change. Analysis of the simulation confirmed that the change was a result of unphysical expulsion of hydrocarbon chain from the interfacial region caused by the too-high biased potential added. This indicated the need to account for the local behaviour of the liquid hydrocarbon molecules. Indeed, the bias potential became sufficiently high to expel hydrocarbon molecules from the interfacial layer. The FEP reconstructed from the WT-metaD simulation shows the same qualitative behaviour as the one obtained within the umbrella sampling (US)/adiabatic biased molecular dynamics (ABMD) framework reported in the main text, with quantitative differences resulting from numerical artifacts. 


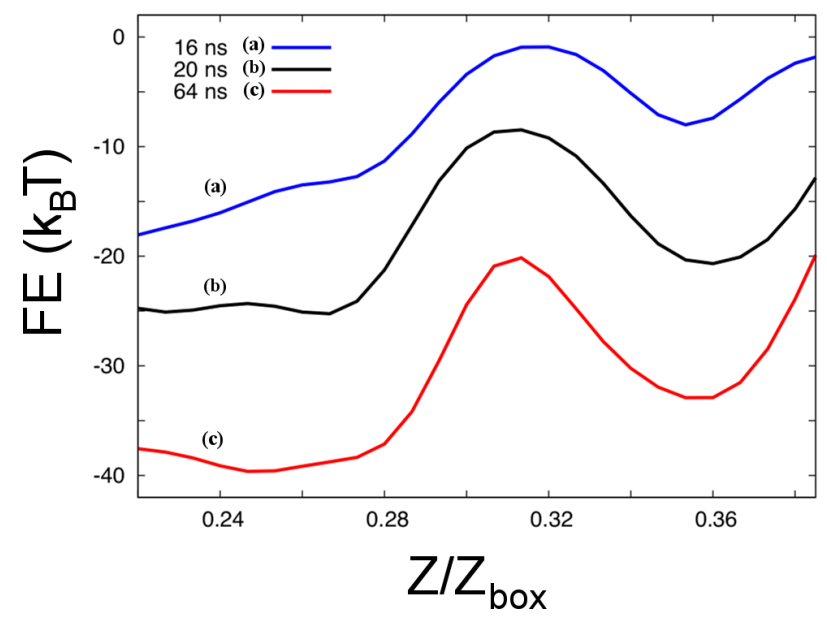

FIG. S1: Reconstructed free-energy profile (FEP), along the minimal pathway as a function of time. The profile was reconstructed after $16 \mathrm{~ns}$ (a), 20 ns (b), and $64 \mathrm{~ns}$ (c). The $x$-axis corresponds to the $Z$-Cartesian coordinate of methane expressed in reduced units, $Z / Z_{\text {box }}$, with $Z_{\text {box }}$ the size of the simulation box along the $Z$ direction.

Umbrella sampling simulations. Once the possible pathways across the AA film were identified, the potential of mean force (PMF) along them was rigorously calculated using US [S4, S5], with the $Z$ - Cartesian coordinate along the pathways as CV. The system was free in the $X-Y$ plane. To design the US windows, the ABMD framework was used [S6 S9]. To reconstruct the minimal and intermediate FEPs discussed in the main text, the authors generated 18 and 33 US windows, respectively, allowing sufficient overlap between adjacent windows. Upon completion of the US simulations, FEPs were calculated from the final 4 ns of simulation time using the weighted histogram analysis method (WHAM) S10]. Statistical error analysis was conducted using the integrated Monte Carlo bootstrapping framework [S11], implemented in WHAM, using 100 resampling trials.

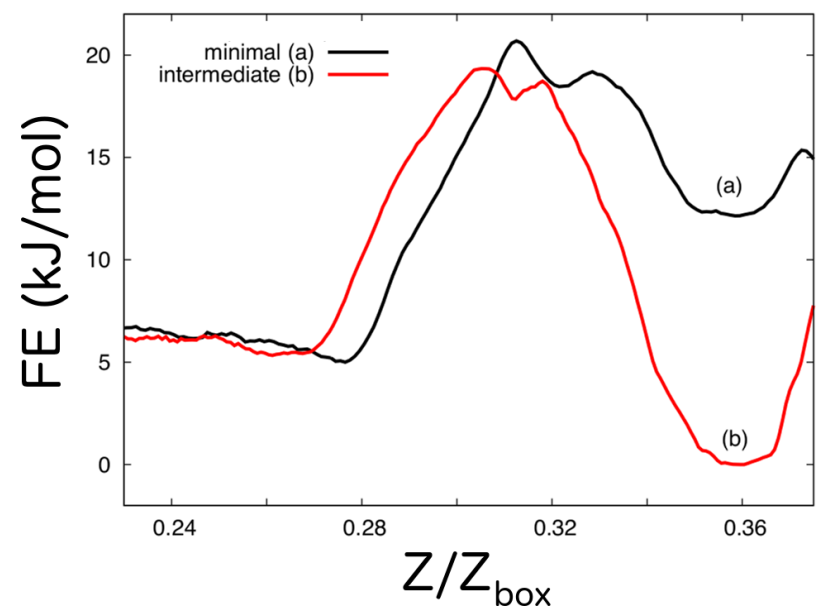

FIG. S2: FEPs associated with the minimal (a) and intermediate (b) pathways, obtained within the US/ABMD framework. The AA layer is flexible, although the central nitrogen atom of each AA molecule remains fixed. The $x$-axis corresponds to the $Z$-Cartesian coordinate of methane expressed in reduced units, $Z / Z_{\mathrm{box}}$.

Figure S2 compares the FEPs discussed in the main text and obtained within US/ABMD frameworks. The activation energies of capture and escape are $\approx 16$ and $\approx 9 \mathrm{~kJ} / \mathrm{mol}$, respectively, for the minimal $\mathrm{FEP}$, and $\approx 14.5$ and $\approx 20 \mathrm{~kJ} / \mathrm{mol}$, respectively, for the intermediate FEP. 


\section{DYNAMICAL ANALYSIS}

Kramers theory. The authors estimated the transition rates along the FE pathways within the Kramers theory framework $\underline{\mathrm{S} 12} \mathrm{S17}]$. In the strong friction regime of interest here, the reaction rate, $k$, is given as $\mathbf{S 1 6}$ ]:

$$
k=\frac{\omega_{0} \omega_{T S}}{2 \pi \gamma} e^{-\Delta F / k_{B} T},
$$

where $\gamma=6 \pi \eta R / m$ is the friction coefficient, with $\eta$ the effective viscosity, and $\Delta F$ the height of the FE barrier. The parameters $\omega_{0}$ and $\omega_{T S}$ represent the stiffness of the potential well and the barrier, respectively, when modeled as parabolic potentials:

$$
V(q)=V_{T S}-\frac{1}{2} m \omega_{T S}^{2}\left(q-q_{T S}\right)^{2}
$$

The nonlinear least-squares Marquardt-Levenberg algorithm was implemented to fit the parameters $\omega_{0}$ and $\omega_{T S}$, as shown in Figure S3, left panel. We obtained $\omega_{T S} \approx 230 \times 10^{13} s^{-1}$ and $\omega_{0} \approx 100 \times 10^{13} s^{-1}$ for the minimal FE pathway, and $\omega_{T S} \approx 100 \times 10^{13} \mathrm{~s}^{-1}$ and $\omega_{0} \approx 130 \times 10^{13} \mathrm{~s}^{-1}$ for the intermediate FE pathway. Figure \$3 right panel, shows an added external potential at $Z / Z_{\mathrm{box}} \approx 0.25$, similar to the $\mathrm{FE}$ barrier observed for $Z / Z_{\mathrm{box}} \in[0.27,0.31]$, to quantify the characteristic time scale for methane capture along the minimal FE path.
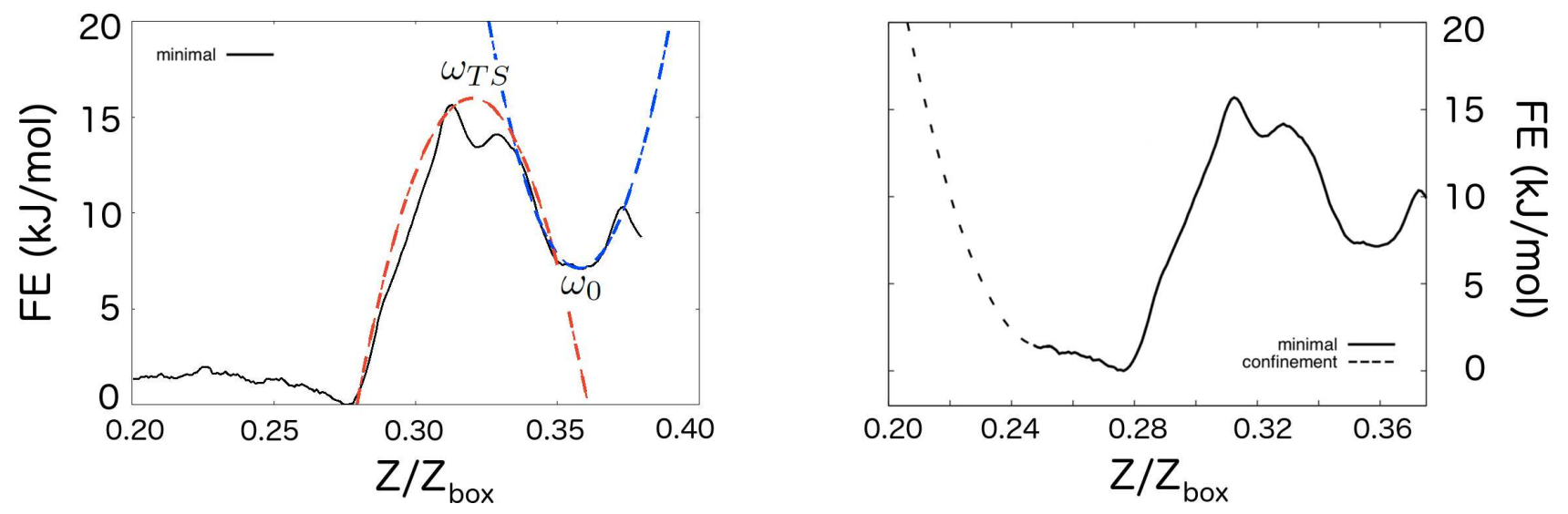

FIG. S3: Fitting of the FEP within parabolic potentials to extract parameters for the Kramers theory (left). Fitting yields $\omega_{0} \approx 100 \times 10^{13} s^{-1}$ and $\omega_{T S} \approx 230 \times 10^{13} s^{-1}$. Representation of the external potential (black dashed line) used in the calculation of the characteristic time scale for methane capture (right).

Analysis of the transition times. Building on the recent method of Parrinello, Salvalaglio, and Tiwary [S18, S19], we extended the metaD scope to assess numerically the characteristic time scales for methane escape and capture. We denote by $\tau$ the physical mean transition time for the methane to pass over the energy barrier, and by $\tau_{M}$ the mean transition time obtained from the WT-metaD run. The latter is linked to the physical mean transition time, $\tau$, by means of the acceleration factor

$$
\alpha(t)=\tau / \tau_{M}=\left\langle e^{\beta V_{\text {bias }}(s, t)}\right\rangle_{M},
$$

where the angular brakets denote an average over a WT-metaD run confined to a metastable basin, and $V_{\text {bias }}(s, t)$ is the WT-metaD time-dependent bias defined in Eq. S1. Because we are not interested in the diffusive converged limit of the metaD simulation, we increased the time lag between two successive Gaussian depositions in the WT-metaD framework, $\tau_{G}=400 \mathrm{ps}$ S18, S19]. We ran 50 independent simulations, considering initial configurations either in the basin at $Z / Z_{\mathrm{box}} \approx 0.36$ (escape rate), or at $Z / Z_{\mathrm{box}} \approx 0.27$ (capture rate). We stopped the simulation when the system crossed the transition state region $\left(Z / Z_{\mathrm{box}} \in[0.31,0.33]\right)$. To assess the reliability of the choice of the CV for the US simulations, we checked that no bias potential was added to the transition state region during the WT-metaD simulations [S19]. We also performed statistical analysis of the distribution of transition times. We performed a twosample Kolmogorov-Smirnov (KS) test, which does not require a priori knowledge of the underlying distribution [S19]. We tested the null hypothesis that the sample of transition times extracted from the metaD simulations and a large 
sample of times randomly generated according to the theoretical exponential distribution reflect the same underlying distribution. The null hypothesis is conventionally rejected if the $p$-value $<0.05$. The KS test has been performed as implemented in the software cran- $\mathrm{R}[\underline{\mathrm{S} 20}]$. The results are reported in the main text.

* Corresponding author: francois.sicard@free.fr.

[S1] A. Barducci, G. Bussi and M. Parrinello, Phys. Rev. Lett. (2008), 100, 020603.

[S2] J. Dama, M. Parrinello and G. Voth, Phys. Rev. Lett. (2014), 112, 240602.

[S3] A. Laio and F. Gervasio, Rep. Prog. Phys. (2008), 71, 126601.

[S4] G. Torrie and J.P.Valleau, J. Comput. Phys. (1977), 23, 187-199.

[S5] J. Kastner, WIREs Comput. Mol. Sci. (2011), 1, 932-942.

[S6] E. Paci and M. Karplus, J. Mol. Biol. (1999), 288, 441-459.

[S7] M. Marchi and P. Ballone, J. Chem. Phys. (1999), 110, 3697-3702.

[S8] C. Camilloni, R. Broglia and G. Tiana, J. Chem. Phys. (2011), 134, 045105.

[S9] F. Sicard and A. Striolo, Faraday Discuss. (2016), 191, 287-304.

[S10] A. Grossfiled, WHAM: the weighted histogram analysis method, version 2.0.9. http://membrane.urmc.rochester.edu/content/wham.

[S11] J. Hub, B. de Groot and D. van der Spoel, J. Chem. Theory Comput. (2010), 6, 3713-3720.

[S12] H. Kramers, Physica (1940), 7, 284.

[S13] P. Hanggi, P. Talkner and M. Borkovec, Rev. Mod. Phys. (1990), 82, 251-341.

[S14] E. Pollak and P. Talkner, Chaos (2005), 15, 026116.

[S15] H.-X. Zhou, Quaterly Rev. Biophys. (2010), 43, 219-293.

[S16] B. Peters, Reaction Rate Theory and Rare Events, 1st Ed.; Elsevier: Amsterdam, The Netherlands, 2017.

[S17] F. Sicard, arXiv:1803.03490 [cond-mat.stat-mech] (2018).

[S18] P. Tiwary and M. Parrinello, Phys. Rev. Lett. (2013), 111, 230602.

[S19] M. Salvalaglio, P. Tiwary and M. Parrinello, J. Chem. Theory Comput. (2014), 10, 1420-1425.

[S20] The Comprehensive R Archive Network. https://cran.r-project.org/ (accessed January 17, 2018) 